\title{
Abu Ali Al-Hasan Ibn Al-Hasan Ibn Al-Haytham, dit Alhazen
}

Mathématicien et astronome Arabe, symbole scientifique de l'an 1000, Alhazen est considéré comme le premier physicien moderne et il est, avec le Grec Claude Ptolémée, l'un des pères fondateurs de l'optique. II a notamment fait avancer le débat millénaire sur la nature de la lumière, et on lui doit le mécanisme de la vision. Surnommé Le Physicien, Alhazen a exercé une influence considérable en Europe médiévale par ses commentaires éclairés des œuvres des auteurs antiques, comme Aristote ou Euclide, et également par ses propres découvertes.

A bu Ali Al-Hasan Ibn Al-Hasan Ibn AlHaytham naît en 965 à Bassorah, ville portuaire située sur le Chatt-el-Arab, estuaire commun des fleuves Tigre et Euphrate, dans la province Irakienne de l'empire Bouyides. Loin de la splendide et extravertie Bagdad, mégapole économique, intellectuelle et artistique, qui abrite alors un million d'habitants à cinq cents kilomètres au Nord, Bassorah est une ville sous domination chiite, qui promeut le savoir et l'enseignement. C'est dans cette atmosphère sereine et éclairée que grandit Al-Haytham, que les générations à venir surnommeront parfois Al-Basri (en référence à sa ville natale) et plus souvent Alhazen (version latine de Al-Hasan).

Alhazen semble n'avoir développé un intérêt marqué pour les sciences que tardivement, s'étant d'abord orienté vers l'étude des textes et de la pensée islamiques. Durant ses premières années, il se consacre à ce que l'on qualifierait aujourd'hui de service civil, sous la forme d'un ministère religieux dans la région de Bassorah. Plus tard, apparemment déçu par les divergences qu'il perçoit entre les différents courants de pensée, progressivement convaincu que seules les sciences peuvent satisfaire sa quête de la vérité et étancher sa soif de comprendre, il quitte son ministère et se plonge avidement dans l'œuvre des penseurs antiques. II trouve apparemment dans la lecture d'Aristote un terreau fertile, et se bâtit très vite une solide réputation de maître du savoir et de mathématicien. Cette notoriété grandissante dépasse les frontières de l'empire Bouyides, et parvient aux portes de l'Égypte.

\section{Un barrage sur le Nil}

Il est utile de rappeler que depuis 969 la dynastie des Fatimides s'est installée dans la vallée du Nil, et y a fondé la ville du Caire, à quelques kilomètres de l'antique Fostat. La jeune cité devient la capitale du califat. En 996, le jeune Al-Hakim bi-Amr Allah, âgé d'à peine 11 ans, accède au trône. II s'avère un despote excentrique et parfois cruel ; mais c'est également un mécène éclairé, qui s'entoure de savants et notamment d'astrologues réputés. II fait construire un observatoire dans son palais, surplombant Le Caire, de même qu'une bibliothèque en I'an 1005, la Maison du Savoir, qui rivalise avec la fameuse Maison de la Sagesse fondée à Bagdad 150 ans plus tôt.

Alhazen, enthousiaste et particulièrement confiant dans la toute-puissance de l'outil mathématique qu'il découvre et manipule de plus en plus habilement, soutient que I'on pourrait régulariser les crues du Nil en utilisant un système de barrages. C'est une information essentielle et une source d'espoir pour le jeune calife Al-Hakim, car les inondations ravagent régulièrement les rives du Roi des fleuves. Il invite Alhazen à le rejoindre, et lui confie une équipe d'ingénieurs et de bâtisseurs. Nous sommes alors en l'an 1010. Alhazen, âgé de 45 ans, et sa petite troupe remontent lentement le cours du Nil vers le Sud, jusqu'à la première cataracte, près d'Assouan. C'est un voyage long : d'abord, une marche forcée sur près d'un millier de kilomètres ; puis, une fois sur place, les repérages et les relevés géologiques. Petit à petit, l'impossibilité de la tâche s'impose à leur esprit, et la ferveur du départ cède le pas à la rude réalité du terrain : Assouan est, sans doute, le lieu propice au grand ouvrage qu'ils projettent, mais leur ingénierie est encore trop rudimentaire ; leur science et leur savoirfaire, pourtant considérables, sans doute alors les meilleurs au monde, ne suffiront pas à apprivoiser le Nil...

II reste à l'annoncer à Al-Hakim. La troupe prend le chemin du retour. Ce sont des hommes lucides, pleinement conscients du risque qu'ils encourent à reconnaître une défaite qui va décevoir le calife. Pour échapper à des représailles despotiques et probablement meurtrières, Alhazen décide de feindre la folie. Fort heureusement, le stratagème fonctionne : Al-Hakim lui laisse la vie sauve mais, pas totalement dupe, il le fait placer en résidence surveillée. Alhazen y reste jusqu'à la mort du calife en 1021. 


\section{Optique et méthode scientifique}

Durant sa captivité Alhazen, jeune chercheur de 50 ans, démarre ses premiers travaux en optique. Fidèle à sa technique, il commence par étudier les grands maîtres : Euclide, Ptolémée, Galien [131-201], sur les travaux desquels il fonde son propre savoir, mais dont il extrapole et élargit considérablement les idées et le cercle de connaissances. Mieux encore, il fonde la méthode scientifique : il base résolument ses recherches et ses nouveaux résultats sur la preuve expérimentale plutôt que sur le raisonnement abstrait, et étaye ses théories par une démarche mathématique. C'est une nouvelle façon de travailler, qui révolutionne la pratique des sciences. II l'applique, avec clarté et pour la première fois, dans son grand œuvre, le Kitab fil Manazir, traité d'optique en sept volumes qu'il rédige de 1015 à 1021. La méthode est convaincante, fertile.

Elle inspirera Robert Grosseteste [1175-1253] et Roger Bacon [1214-1292], qui la diffuseront à leur tour au Moyen Âge en Europe.

Les résultats qu'obtient Alhazen marquent un tournant pour la théorie de la lumière. Son Kitab fil Manazir, traduit en Opticae thesaurus Alhazeni en 1270, occupe dans I'histoire des sciences une place comparable à celle de l'Almageste de Claude Ptolémée et servira d'ouvrage de référence à la plupart des livres d'optique qui seront écrits pendant près de cinq siècles. Et de fait, les innovations sont nombreuses. Pour ses expériences il utilise, pour la première fois, une camera obscura ; il étudie l'effet de grossissement à travers des vases de verre remplis d'eau ; il s'intéresse également à la réfraction, proposant l'idée que la lumière est un mouvement qui admet plusieurs vitesses selon le milieu de propagation (moindre dans un milieu plus dense), mais il n'en détermine pas la loi, qu'il faudra encore six siècles à établir (par Descartes et Snell)... Ses multiples percées en font, sans conteste, le père de l'optique moderne.

Sans surprise, Alhazen se mesure aussi au mystère millénaire du mécanisme de la vision. Deux écoles coexistent depuis l'antiquité : pour Aristote et ses disciples, I'œil reçoit une forme complète en provenance de l'objet observé ; dans l'approche du philosophe grec Empedocles, reprise par Ptolémée et Euclide, et qui fait alors référence, l'œil voit grâce à la lumière qu'il projette lui-même sur les objets alentour. Alhazen souffle un air de modernité et propose la première explication correcte du processus de la vision, démontrant par l'expérience que la lumière est réfléchie par l'objet vers l'œil qui en forme alors une image inversée. Ce travail mènera plus tard Kepler au mécanisme de la formation des images. Allant plus loin, Alhazen montre, à l'aide d'arguments géométriques, comment la vision binoculaire permet d'appréhender les distances à l'objet vu, de même que les dimensions de cet objet.

Dans son Mizan al-Hikmah, Alhazen disserte enfin sur la densité de l'air et émet l'hypothèse que l'atmosphère a une hauteur finie. Il en déduit la lumière crépusculaire, celle que l'on perçoit par réfraction lorsque le soleil, encore invisible, est à moins de $19^{\circ}$ sous I'horizon.

\section{Travaux mathématiques}

En mathématicien averti, Alhazen s'intéresse à l'analyse, utilisée par les anciens Grecs pour la résolution de problèmes géométriques, et qu'il extrapole au traitement d'équations algébriques. Il développe ainsi les concepts de géométrie analytique.

\section{घபlletin d’abunnement}

QUANTITÉ $\quad$ TOTAL

Abonnement (6 numéros)

$\square$ Tarif normal : $\square$ France : $60 €$ TTC $\bullet \square$ UE, Suisse : $74 €$ TTC $\bullet \square$ Autres pays : $80 €$ TTC

$\square$ Étudiants (à titre individuel, sur justificatif) $38 €$ TTC

Commande de numéros déjà parus (10€ par exemplaire)

Indiquer ci-dessous le numéro de la revue commandée et, en dessous, la quantité souhaitée.

ex:

$\mathrm{PH} 40 \mathrm{PH}$

$\mathrm{PH}$

$\mathrm{PH}$

$\mathrm{PH}$

PH

$\mathrm{PH}$

3

TOTAL $\square$ Je joins un chèque de $\square$ Je règle par carte bancaire Date de fin de validité $L$
Numéro de carte

euros à l'ordre d'EDP Sciences

Cryptogramme visuel (Code inscrit au dos de ma carte) $L+1$ $\square$ Je préfère régler par chèque, à réception de facture (uniquement entreprises et institutions)

$\square$ Merci de me faire parvenir une facture acquittée

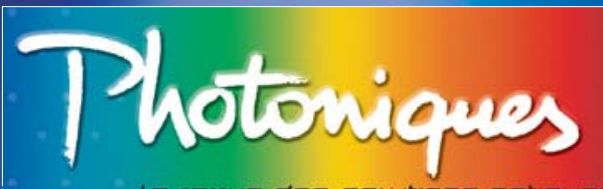

a revue des salutians aptipues

Photoniques $n^{\circ} 48$ - septembre-octobre 2010 Photoniques $n^{\circ}$ 49/50 • novembre-décembre 2010 Photoniques $n^{\circ} 51 \quad \bullet$ janvier - février 2011 Photoniques $n^{\circ} 52 \quad \bullet$ mars-avril 2011 Photoniques $n^{\circ} 53 \quad \bullet$ mai-juin 2011 Photoniques $n^{\circ} 54 \quad$ • juillet-août 2011

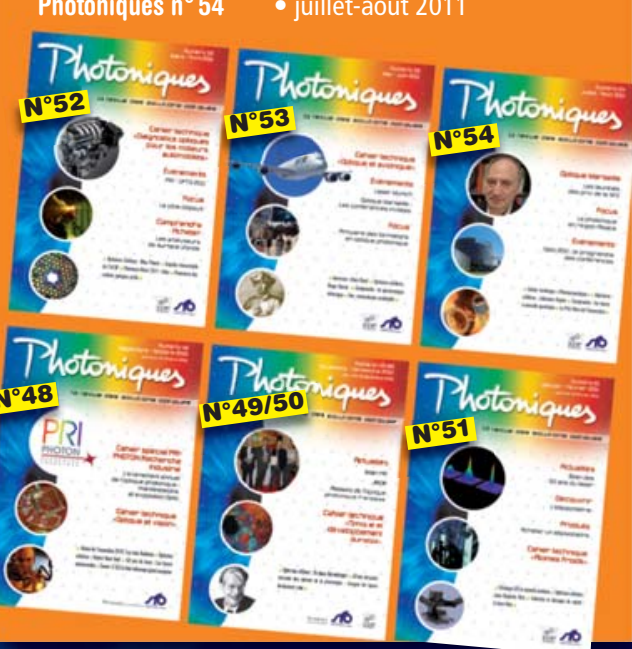




\section{QPTICIENS CÉLĖBRES}

Les liens avec l'optique restent omniprésents : dans le livre $V$ du Kitab fil Manazir consacré à la catadioptrique, il pose une question (le fameux problème du billard d'Alhazen) que l'on peut énoncer ainsi : étant donné deux billes $A$ et $B$ placées en deux points quelconques d'un billard circulaire, déterminer le point d'impact de la bille $A$ sur le rebord pour qu'elle heurte la bille $B$ après un seul rebond. Cette question, que l'on sait traiter aujour$\mathrm{d}$ 'hui en résolvant une équation du quatrième degré et dont les connexions avec l'optique sont évidentes, semble avoir interpellé d'autres esprits depuis l'antiquité. Lui-même en propose une solution géométrique. De Vinci et Huygens, plus tard, s'attaqueront aussi au problème.

Après la mort du calife Al-Hakim en 1021, Alhazen cesse de feindre la folie et retrouve sa liberté de déplacement. À l'âge de 56 ans, il peut enfin quitter l'Égypte et en profite pour entreprendre quelques voyages, notamment en Espagne. Mais c'est un périple qui le ramène définitivement au Caire. Par la suite, Alhazen ne quitte plus les alentours de la mosquée Al-Azhar et I'université attenante, la Maison du Savoir, riche de nombreux livres de chimie, d'astronomie et de philosophie, en plus des textes strictement religieux. Dans cet environnement propice, il rédige plusieurs traités de mathématiques, enseigne et poursuit ses recherches scientifiques.

Alhazen décède au Caire, à la fin des années 1030, au sommet de sa gloire.

\section{Héritage}

Bien qu'arrivé tardivement dans le monde des sciences, Alhazen a produit près d'une centaine d'ouvrages, dont près de la moitié nous est parvenue, notamment grâce aux traductions latines. Son œuvre, même selon les critères modernes, est extraordinairement éclectique et prolifique. Il a étudié et proposé des percées remarquables dans des domaines aussi variés que l'optique, incluant la théorie de la lumière et celle de la vision, l'astronomie, la médecine, les mathématiques en allant de la géométrie à la théorie des nombres. Son influence sera largement reconnue au cours des siècles, et son apport tenu en haute estime. Il forme quelques disciples, dont certains le côtoient pendant plusieurs années, mais son héritage est surtout fertilisé par les générations suivantes. Ainsi son œuvre est discutée par le philosophe et médecin andalou Ibn-Ruchd (Averroès) [1126-1198], reprise en détail et enrichie par le grand scientifique perse Kamal al-Din al-Farisi [1267-1320] qui rédigera le Kitab Tanqih al-Manazir. Ses idées diffuseront ensuite en Europe grâce au De Perspectiva du moine Vitellion [1230-1280]. Roger Bacon participe à son tour au relais planétaire du savoir en fondant ses propres travaux d'optique sur le Kitab fil Manazir.

\section{Riad HAIDAR \\ Onera}

haidar@onera.fr

\section{Références}

[1] Alhazen, John J. O'Connor et Edmund F. Robertson. MacTutor History of Mathematics. Université de St Andrews.

[2] L'âge d'or des sciences arabes. Ahmed Djebbar (Éditions Le Pommier, 2005).

\section{$\square$ Monsieur}

$\square$ Madame

$\square$ Mademoiselle Société

Adresse complète (précisez BP, Cedex...).

Code postal

Téléphone

E-mail

\section{Nom}

Prénom

Pour que nous puissions adapter Photoniques aux besoins de ses lecteurs, merci de bien vouloir nous indiquer :

\section{Votre service :}

$\square$ Direction générale

$\square$ Conseil. Marketing. Publicité

$\square$ Études. Développement

$\square$ Méthodes. Ordonnancement

$\square$ Recherche

$\square$ Contrôle

$\square$ Production

$\square$ Entretien. Sécurité

$\square$ Achats

$\square$ Enseignement. Formation

$\square$ Autre :

\section{Votre fonction :}

$\square$ Chef d'entreprise. DG

$\square$ Directeur

$\square$ Chef de service

$\square$ Ingénieur

$\square$ Chef de projet

$\square$ Technicien

$\square$ Agent de maîtrise

$\square$ Chercheur

$\square$ Directeur de recherche

$\square$ Universitaire (enseignant, chercheur)

$\square$ Autre
Service..

Pays

Fax

Nombre de salariés :

$\square$ 1-9

$\square$ 10-49

$\square$ 50-99

$\square$ 100-199

$\square$ 200-499

$\square 500$ et +

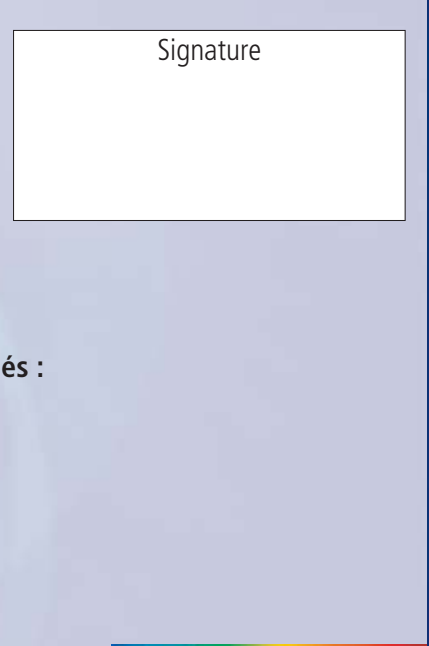

